\title{
Sequencing microbial genomes - what will it do for microbiology?
}

\author{
P. J. JENKS
}

Unité de Pathogénie Bactérienne des Muqueuses, Institut Pasteur, 25-28 Rue de Dr Roux, 75724 Paris, Cedex 15, France

\begin{abstract}
In 1995, Haemophilus influenzae became the first free-living organism to have its entire genome sequence published. Since then, many similar projects have been started and, by the millennium, the genomes of a significant number of important human pathogens will have been sequenced. During this period of increasing access to microbial sequence data, parallel advances have occurred in techniques that allow the large-scale study of the entire genetic complement of micro-organisms. In the near future, these approaches will enable researchers to unravel further the complexity of microbial pathogenesis and identify new virulence determinants. Many of these will be suitable targets for development as diagnostic reagents, antimicrobial agents and vaccine candidates. Although it is difficult to predict the full impact that this almost overwhelming volume of information will have on the practice of microbiology, it is clear that it will result ultimately in new ways of diagnosing and combating infectious diseases.
\end{abstract}

\section{Introduction}

The study of infection is being revolutionised by the development of technology that allows the sequencing and analysis of entire microbial genomes. The genomes of a number of important human pathogens have already been or are in the process of being sequenced, and the status of these projects is shown in Table 1. The continuing release of data from these projects, combined with advances in techniques used to investigate molecular pathogenesis, provides a frame- work that will facilitate our understanding of these organisms and, ultimately, lead to new anti-infective strategies. This review aims to give some background to the sequencing methods used, to describe how sequence information can be accessed and analysed, and to discuss some of the novel approaches that utilise the information generated by these projects. In general, the discussion will be limited to human pathogens.

Until recently, the technology required for large-scale microbial sequencing projects was not available and

Table 1. Current status of genome sequencing of important human pathogens*

\begin{tabular}{lll}
\hline Completed genome sequences & Genome sequences in progress & Potential future sequencing projects \\
\hline Haemophilus influenzae & Actinobacillus actinomycetemcomitans & Campylobacter jejuni \\
Mycoplasma genitalium & Aspergillus nidulans & Brucella abortus \\
Mycoplasma pneumoniae & Bacillus subtilis & Haemophilus ducreyi \\
Helicobacter pylori & Chlamydia trachomatis & Mycobacterium avium \\
Borrelia burgdorferi & Enterococcus faecalis and faecium & Pasteurella multocida \\
Escherichia coli K-12 & Legionella pneumophila & Porphyromonas gingivalis \\
Streptococcus pneumoniae & Leishmania major & Salmonella serotype Typhimurium \\
Treponema pallidum & Mycobacterium leprae & Trypanosoma brucei \\
Ureoplasma urealyticum & Mycobacterium tuberculosis & \\
& Neisseria gonorrhoeae and meningitidis & \\
& Plasmodium falciparum & \\
& Pseudomonas aeruginosa & \\
& Rickettsia prowazekii & \\
& Staphylococcus aureus & \\
& Streptococcus pyogenes & \\
& Trypanosoma cruzi & \\
& Vibrio cholerae & \\
\hline
\end{tabular}

${ }^{*}$ Compiled from the MAGPIE project web site (http://www.mcs.anl.gov/home/gaasterl/magpie.html). 
early attempts were limited to organisms with relatively small genomes, such as the bacteriophages $\phi \mathrm{X} 174$ and $\lambda[1,2]$. A number of viruses were also sequenced, including cytomegalovirus, vaccinia and variola smallpox virus, the latter being the first to be completely sequenced with automated technology [3-5]. One major constraint to the early projects was the need to create an ordered library of the genome before sequencing could begin. This was extremely time-consuming and consisted of assembling an ordered set of overlapping fragments or clones that together represented the entire genome of the organism. By means of a procedure known as 'shotgunning', each clone was then broken into random smaller fragments which were sequenced. These were re-assembled by overlapping regions to give the sequence of the original clone and, once this had been repeated for all the clones in the library, the sequence was complete.

The new, more rapid approach is to 'shotgun' the entire bacterial chromosome to create a random DNA fragment library. These unordered fragments are sequenced by banks of automated sequencers and reassembled in the correct order with powerful computer software. A sufficient number of fragments is sequenced to ensure adequate coverage of the chromosome, so that when they are assembled, most of the genome is represented without leaving too many gaps (to achieve this the entire genome is usually covered 5-8-fold, leaving $c .0 .1 \%$ of the total DNA unsequenced). Although this sequencing and assembly of unselected pieces of DNA eliminates the need for initial mapping and is faster, it relies on enormous computational power to re-assemble the huge number of fragments once they have been sequenced $[6,7]$. After the random fragments have been assembled by areas of overlapping sequence, any remaining gaps can be identified and closed. Sophisticated data processing then permits detailed annotation of the data and prediction of putative coding regions, operons and regulatory sequences.

These advances in automated DNA sequence analysis and bio-informatics led to the publication of the entire genome sequence of Haemophilus influenzae strain $\mathrm{Rd}$ in July 1995 by The Institute for Genomic Research (TIGR) [8]. This organism was sequenced and annotated in $<12$ months with an error rate of one base in $(5-10) \times 10^{3}$ and an estimated cost of 33 pence $(\$ 0.5) /$ base. New approaches to sequencing strategy and algorithms for assembling data are appearing constantly, and sequencing is seen as a rapid and cost-effective way of accessing biological data. Several projects for sequencing a wide range of micro-organisms have subsequently been started with funding from governmental agencies, medical charities and private industry. A number of international collaborations have been established to tackle the larger genomes, e.g., the yeast Saccharomyces cerevi- siae and the 14 chromosomes of Plasmodium falciparum. It is likely that the genomes of a large number of important human pathogens will be sequenced fully by the millennium (Table 1).

This rapid expansion in microbial genome sequencing is not without problems, not least the fact that data from such projects have not always been published or deposited in public data banks. However, significant advances have been made recently in this area, and an increasing amount of information is now available publicly through readily accessible databases (see below). Another highlighted problem has been that of 'redundant genome sequencing', where certain organisms, including Mycobacterium tuberculosis, Staphylococcus aureus and Helicobacter pylori, have been sequenced partially by more than one organisation [9]. The choice in certain cases to sequence strains of unknown or low virulence (such as the laboratory strain of $M$. tuberculosis (strain H37Rv) and Escherichia coli strain K-12 which contain few of the organism's virulence factors) has also been criticised [10]. Hopefully, improved communication between sequencing groups and researchers will limit these problems in the future. Another concern is that the current rush to release data may compromise its accuracy, particularly in view of the lack of quality control on data entering public databases. This extends to the accuracy of annotation and the allocation of biological function solely on the basis of raw sequence data. It is easy to overlook the fact that these everexpanding volumes of computer-generated data still require classical biological and physiological study before definitive functional analysis of an organism can be said to be complete.

\section{Accessing microbial sequence information}

Keeping up with developments in microbial sequencing projects can be a major problem, despite the fact that data are often available through public databases, many of which are updated on a daily basis. A number of extremely useful sites exist on the Internet that allow ready access to this information, and these are usually designed in a user-friendly way. A selection of the sites used regularly by the author is given in Table 2. As well as giving details of sequencing projects, these also provide analytical software and links to other sites of potential interest. Such a powerful network allows rapid access to enormous amounts of information and represents a tremendous resource for research and teaching.

An excellent place to start is the Magpie Genome Sequencing Project List, maintained and updated by Terry Gaasterland of the Argonne National Laboratory. This site is well designed and contains a comprehensive list of current genome sequencing projects with individual summaries. A series of analytical tools for 
Table 2. Internet sites useful for accessing and utilising microbial genome databases*

\begin{tabular}{ll}
\hline URL & Notes \\
\hline $\begin{array}{l}\text { General resources } \\
\text { http://www.mcs.anl.gov/home/gaasterl/magpie.html } \\
\text { http://www.pasteur.fr }\end{array}$ & $\begin{array}{l}\text { MAGPIE project } \\
\text { http://www.qmw.ac.uk/ rhm001/index.html }\end{array}$ \\
$\begin{array}{l}\text { The Pasteur Institute home page } \\
\text { Specific sequencing projects } \\
\text { http://www.tigr.org }\end{array}$ & $\begin{array}{l}\text { The Institute for Genomic Research (Haemophilus influenzae, Mycoplasma } \\
\text { genitalium, Helicobacter pylori) }\end{array}$ \\
http://www.sanger.ac.uk/Projects & Sanger Centre (Mycobacterium tuberculosis, Mycobacterium leprae, \\
http://www.genome.ou.edu & Plasmodium falciparum, Neisseria gonorrhoeae) \\
& University of Oklahoma (Neisseria gonorrhoeae, Streptococcus pyogenes, \\
& Actinobacillus actinomycetemcomitans, Aspergillus nidulans) \\
Further resources/information & \\
http://www.seqnet.dl.ac.uk//home.html & SEQNET at Daresbury \\
http://www.ncbi.nlm.nih.gov & National Center for Biotechnology Information \\
http://www.ai.sri.com/ecocyc/hincyc.html & Encyclopaedia of H. influenzae genes and metabolism \\
http://www.ai.sri.com/ecocyc/ecocyc.html & Encyclopaedia of E. coli genes and metabolism \\
http://www.pdb.bnl.gov & Protein Data Base \\
\hline
\end{tabular}

${ }^{*}$ All sites have been visited or used by the author.

assembling and evaluating sequence information, plus links to other relevant databases, are also available, as is access to various metabolic and functional pathways. Other useful general resource sites include the Pasteur Institute's home page (with a version in English), and the Microbial Underground! which is based at Imperial College. As well as providing access to sequence databases and sequence analysis software, these sites also allow cross-reference to electronic journals and related sites of interest in general microbiology, medicine and molecular biology.

To obtain information on specific micro-organisms, it is usually necessary to access the organisation responsible for each individual project. The TIGR web site gives information on their key sequencing projects, and analysis of the databases can be achieved in a very easy, user-friendly way. Also provided are software tools for use by the scientific community, information on the laboratory methods used, and links to other genomic centres, databases and electronic journals. Similar information on other organisms can be found at the Sanger Centre home page.

Through GenBank, the National Center for Biotechnology Information (NCBI) acts as the main curator of microbial genomic information. The NCBI site allows database searches, full analysis of microbial sequences, and also connects to biologically related sites. For those requiring specialised programs for the analysis of sequence data, SEQNET provides a selection of analytical software tools plus instructions and help on how to use them. Structural analysis of predicted gene products can be obtained through the Protein Data Bank, which contains an updated archive of the 3-D structures of biological macromolecules. A more detailed functional analysis of sequenced organisms is also possible, for example, by using sites dedicated to the genes and metabolism of $H$. influenzae and $E$. coli. The use of such computerbased techniques to analyse sequence data has already led to some important breakthroughs in the study of pathogenic bacteria.

\section{Microbial sequence data and the study of infection - present status}

There are already several examples of how rapidly novel biological information can be accessed once the complete genome sequence of an organism is available. The simplest method is to perform homology searches in existing databanks to identify recognised virulence determinants in new species. This approach has been used to study the genetics of lipopolysaccharide (LPS) biosynthesis in $H$. influenzae [11]. When the genome sequence of this bacterium was released, seven enzymes had been implicated in the synthesis of LPS, a molecule that is both a major virulence determinant and a target for the host immune response. By searching the complete sequence of $H$. influenzae for LPS genes known to exist in other bacteria, a further 25 candidate genes were identified. The construction of defined mutants confirmed a potential role for most of these genes in LPS biosynthesis, and virulence studies in the infant rat model have enabled the minimal LPS structure required for intravascular dissemination to be predicted. It is hoped that extension of this work will identify conserved motifs that are potentially useful as vaccine candidates for non-type $b$ strains.

Searching for candidate virulence genes by the identification of recognised regulatory sequences is another example of how microbial sequence information can be used [12]. In $H$. influenzae, a number of cell surface molecules, including LPS, undergo slight changes of structure that allow the organism to adapt to micro-environmental changes and evade the host's immune system (a process known as phase variation). It was known that the regulation of phase variation of LPS was mediated by the loss or gain of repeating 
units in the regulatory region of the LPS genes. This resulted in altered reading of these genes, and hence changes to the exposed structural molecule. It was postulated that other genes potentially important in mucosal colonisation might also use this strategy, and, therefore, the $H$. influenzae genome was searched for other examples of these repeating units. The search revealed similar multiple repeats at nine new locations, and most of the genes controlled by these regulatory sequences were found to be virulence factors, including a gene involved in LPS biosynthesis $(\lg t G)$, one that resembled an adhesin, and a homologue of a haemin-binding receptor [12].

Many important virulence genes of pathogenic bacteria are often grouped together in genetic elements termed pathogenicity islands (PAIs) [13]. These discrete segments of DNA usually have a different overall nucleotide composition from the rest of the chromosome, and it has been demonstrated that novel PAIs can be identified by scanning a complete genomic sequence for such differences with sophisticated computer software [14]. With modern bio-informatics it is also possible to compare entire microbial genomes in order to identify factors unique to certain pathogens, or those that are generally conserved for pathogenicity. Such approaches are also useful in micro-organisms with considerable genetic heterogeneity, such as $H$. pylori. By using the recently sequenced strain as a blueprint, different clinical and geographical isolates can be compared to obtain information on the global genetic make-up of this important pathogen and to identify those factors associated with different clinical outcomes [15]. Access to sequence data of $H$. pylori has already provided more information on the unusual metabolism of this organism and given clues as to how it is able to survive in the uniquely hostile environment of the human stomach. It is hoped that it will also facilitate research into the aspects of the microbe-host relationship that result in persistence and long-term carriage, as well as identifying factors that are important in carcinogenesis. Two other important bacteria that have been completely sequenced are $E$. coli, an organism whose metabolic function, genetics and physiology have been studied extensively [16], and Mycoplasma genitalium, which has the smallest known genome for a self-replicating free-living organism [17].

Although this review concentrates mainly on human pathogens, a number of other micro-organisms have also been sequenced. The release of the sequence of the archaeon Methanococcus jannaschii has provided important information on the molecular basis of evolution [18]. Computational analysis of this genome demonstrated that the Archaea are different from all other living organisms, including bacteria, and confirmed the existence of three separate domains of life (Archaea, Bacteria and Eukarya). Sequencing projects are also under way on a number of organisms that survive in hot springs and deep-sea vents, and others that are able to resist radioactivity or extremes of temperature (the 'extremophiles'). Genetic information on these will offer insights as to how bacteria are able to survive in such harsh environments, and may assist the discovery of novel enzymes suitable for use in research, health care and industry.

\section{Microbial sequence data and the study of infection - future prospects}

One feature of the microbial genomes that have so far been sequenced is that a significant proportion (40$60 \%$ ) of the predicted coding regions have been without known function at the time of publication. Although the number of these so-called 'orphans' has been subsequently reduced by rigorous databank searching, the challenge of assigning function to these currently unclassified genes is now a major research priority. In the past, the usual approach to the functional assessment of a gene was to create an isogenic mutant and screen for altered (usually loss of) function, a process that was inefficient and not applicable to all organisms. Generation of mutants was done either in a random fashion (e.g., by transposons) or was directed (when the sequence of the gene was known). Directed mutagenesis, which is generally preferable, is obviously easier once an organism's entire sequence is known. However, although mutating and studying individual genes remains a valid approach, investigators are looking increasingly at methods that allow the large-scale analysis of genomic data and that also permit more meaningful work to be performed in vivo. By adopting a more global approach and identifying genes that are expressed preferentially during the infection process, it is hoped that novel targets suitable for development as therapeutic agents will be identified.

\section{Signature-tagged mutagenesis}

As already mentioned, characterising the function of individual genes by mutational inactivation is a process that is inefficient and time-consuming. A more efficient approach is to create a large bank of mutants and study these together. This can be achieved if each one is labelled with a unique identifying 'bar code' or tag. Each tag consists of a short unique segment of DNA integrated into the chromosome of each mutant that enables an individual member of a pool of mutants to be identified readily from the others. This is particularly useful for studies in vivo, as if a mutant is absent after passage through an animal, the tagged mutation it harboured may be in a gene essential for survival. This technique, which allows attenuated clones to be identified readily in a large population of mutants, is known as signature-tagged mutagenesis (STM) [19]. It was developed in the Salmonella serotype Typhimurium mouse model of typhoid and has already identified 
a new pathogenicity island in this organism [20]. In this model, randomly constructed and tagged mutants were pooled, injected into a mouse, and then recovered from the target organ (the spleen). Potentially important clones were identified by comparing the population of tags or 'bar codes' in the pool injected into the animal with those recovered from the target organ. Those with attenuated virulence (and hence with a mutation in a gene potentially important in establishing infection) were not recovered from the animal and were, therefore, absent from the latter population. Although designed originally for random transposon mutagenesis, STM is potentially suitable for use in combination with other mutagenesis techniques. Once the entire sequence of an organism is known, it is relatively straightforward to create directed mutations in a large number of candidate virulence genes and 'bar-code' each one as it is constructed. In theory, it is also possible to identify all the potential coding regions of an organism and sequentially mutate and tag its entire genome, so enabling the global study of genes that are important for colonisation and survival.

By identifying attenuated clones, STM utilises negative selection to identify genes that may be important for pathogenesis. Positive selection for genes induced specifically during host infection is possible by a technique termed in-vitro expression technology (IVET) [21]. This has been used in Pseudomonas aeruginosa to identify genes that are important. for the infection of neutropenic mice [22], and also those induced by respiratory mucus derived from cystic fibrosis patients [23]. Individual genes expressed specifically during infection or by certain environmental stimuli can also be studied by fusion to a 'reporter' that indicates when the gene is 'switched on'. Green-fluorescent protein (gfp) is a newly described reporter that allows genes induced under certain conditions (e.g., within macrophages) literally to 'light-up' and be identified readily [24]. A detailed description of IVET and gfp is beyond the scope of this article, but more detailed information can be found in an excellent recent review [10].

\section{Differential display}

Another way of identifying factors important in the colonisation of a host is to examine patterns of gene expression under different growth conditions or in different models of infection. The specific induction of proteins can be detected by pulse-labelling bacteria with $\left[{ }^{32} \mathrm{~S}\right]$ methionine, exposing the organism to the test condition, and then extracting and resolving the synthesised proteins by two-dimensional SDS-PAGE. Proteins induced under the test conditions are cut out of the gel, subjected to proteolytic cleavage and sequenced by mass spectrometry. Newly developed software is able to analyse the amino acid sequence obtained and find the best match for it in the databases. This approach is easier and more powerful for an organism that has been sequenced completely and allows hypothetical gene products to be confirmed as real proteins. It is also possible to monitor changes in the type and levels of proteins expressed when growth conditions are altered. This global approach to the study of proteins has been used successfully in Salmonella Typhimurium, where proteins important in the invasion of cells were identified by comparing gel patterns after labelling the organisms in the presence and absence of a fixed epithelial cell layer [25]. This system has also offered insights into how $H$. pylori is able to survive and colonise the human stomach by identifying two proteins that are overexpressed when the organism is exposed to an acid environment $[26,27]$.

Changes of gene expression relevant to microbial pathogenesis can also be studied at the mRNA level by a PCR-based method called differential display [28]. After exposure of the organism to a certain environmental condition, such as contact with cultured epithelial cells, the mRNA is extracted. This mixed mRNA population is amplified by PCR with arbitrary primers and the products are resolved on a sequencing gel to give a unique mRNA 'fingerprint'. Comparison of fingerprint profiles generated under different conditions allows the identification of unique, fluctuating bands that represent those mRNAs displayed differentially under these conditions. These can be isolated, sequenced and studied further. This approach has been used to study the response of Salmonella Typhimurium to oxidative stress induced by hydrogen peroxide [29]. Total RNA extracted from hydrogen peroxidetreated and -untreated cultures was amplified with six arbitrary primers and comparison of fingerprints identified a single product that was induced preferentially by oxidative stress. Again, this technique is facilitated if the organism under study has been sequenced, as not only is the databank search of any isolated mRNA sure of finding a match, but it is also possible to design primers that will amplify the entire population of mRNAs for that organism (as all the potential coding regions are known).

\section{The 'DNA chip'}

One emerging technology whose development is ideally suited to the genomic era is that of high density oligonucleotide arrays, or 'DNA chips'. These DNA chips consist of specific oligonucleotide probes that are spotted or directly synthesised in an ordered fashion on to glass or nylon solid supports [30,31]. They are constructed with light-directed oligonucleotide synthesis (an adaptation of a technique used in the computer chip industry) and thousands of these small probes can be fitted on to an area the size of a thumb nail. The availability of microbial sequence data means that the oligonucleotides used on the chip can be designed rationally to include a probe for every gene of an individual organism. It is, therefore, possible to 
design custom-made DNA chips that contain a representative of every gene of, e.g., $H$. influenzae or $H$. pylori, each with its own address on the array. Once synthesised, the DNA chips can be hybridised with fluorescently labelled target sequences and the hybridisation of the target to each miniaturised probe is determined by scanning confocal microscopy [32,33]. This automatically detects and analyses patterns of fluorescence and, because each probe has a specific address, those that are positive can be identified by the co-ordinates of the fluorescent spots on the array. Because so many probes are included on an individual chip, thousands of hybridisations can be performed simultaneously and this, potentially, provides a rapid and cost-effective way to determine gene function, monitor gene expression patterns and screen for gene mutations [34-37].

By constructing an array that contains a probe for each gene of an organism, it is possible to monitor global microbial gene expression under different conditions. This is a similar approach to differential display, but is considerably more powerful. After exposing an organism to different environmental stimuli or passing it through an animal model, the mRNA is isolated, fluorescently labelled and hybridised to the array. Novel mRNAs induced under these conditions will hybridise to the corresponding probes on the array and generate fluorescent spots. The fluorescent patterns generated are assessed and, as each probe is situated at a spatially addressable location on the array, the individual genes turned on by each individual environmental stimulus can be identified. This approach is rapid and quantification is possible as the pattern and intensity of fluorescent signal gives information on the amounts of a particular mRNA present in the mixture analysed. This technique could, potentially, be extended to monitor expression, by a single hybridisation reaction, across the entire genome of selected pathogens. By using animal models of infection, it is also possible to study sequential gene expression during the course of an infection or specific expression at different sites in the host's body.

By using a concept similar to that of STM, DNA chips can also be used for the large-scale parallel analysis of pools of deletion mutants. In this case, the DNA chip is made up of a series of different 20-base segments of DNA that act as 'tag-detectors'. A pool of deletion mutants is constructed and each is labelled individually with a unique 20-base tag sequence. Each of these tags is designed to have one complementary sequence ('tag-detector') somewhere on the array and therefore each mutant can be detected and identified uniquely by hybridisation to this particular chip. Again, the intensity of the hybridisation signal indicates the relative abundance of the corresponding deletion mutant in the pool, and the relative importance of that gene in certain environmental conditions or in the colonisation of an animal model can be assessed. A theoretical example would be in the study of factors important for adhesion. A random pool of mutants is generated, tagged and incubated with an epithelial cell layer. Those with mutations in genes important for adhesion would fail to adhere and would be lost from the pool. After exposure to the cell line, the pool is hybridised to the array and the potential adhesin mutants can be identified as areas of reduced or no fluorescence on the array (as they are no longer present in the pool). The co-ordinates of these 'holes' allow the exact gene(s) involved to be identified. This technique (termed signature tagged allelic replacement or STAR) has already been used successfully in the yeast $S$. cerevisiae, where 11 tagged mutants were grown under different conditions and the survival of each mutant was measured by quantification of fluorescence [36]. This experiment is viewed as a pilot study for the systemic mutational analysis of the complete genome of $S$. cerevisiae as, in theory, it is possible to analyse simultaneously all genes under different conditions with a single hybridisation. The technique can be applied readily to any pathogen and, once a pool of tagged mutants has been constructed, genes important for colonisation and adaptation to the host can be identified readily.

This novel hybridisation approach is also an efficient way to screen small fragments of DNA rapidly and accurately for mutations. If hybridising DNA has the identical sequence to a probe on the chip it will hybridise exactly, whereas if mismatching occurs (because of a mutation) this can be detected as reduced signal intensity with sophisticated software. At present, this is being developed primarily to detect mutations in genes involved in cancer and human hereditary disease $[38,39]$. However, point mutations are important in the development of resistance to many antimicrobial agents, and this technique has already been used to study the changes in the HIV-1 protease gene known to contribute to drug resistance in this virus [37]. In the future, this may provide a rapid way of screening isolates for the presence of recognised and new resistance mechanisms, as well as allowing pathogens to be screened for adaptive mutations in important virulence factors.

\section{Conclusions}

In the near future, the application of new molecular techniques, in combination with the availability of sequence data, will allow the rapid dissection of entire microbial genomes, resulting in an unprecedented understanding of the infection process. This will undoubtedly lead to a fundamental re-appraisal of our view of the relationship between the organism and its host, and of how microbial disease arises. Although there is often a delay between research discoveries and their application to diagnostic and clinical microbiol- 
ogy, the rapid progress currently being made in research is soon likely to have an effect in these areas. Analysis of the genomes of pathogenic micro-organisms will lead inevitably to the discovery of novel and important virulence determinants. The increasing ability to dissect out those genes that are essential for viability, or that are expressed during the infection process, is likely to provide suitable molecular targets for future antimicrobial design. The fact that it has been almost 30 years since a new class of antibiotic was discovered, combined with the increasing problem of antimicrobial resistance, means that such novel approaches are extremely timely. These focused genomic-based approaches also allow the study of the complete antigen complement of an organism and offer unprecedented opportunities for discovering new vaccine candidates.

Microbial genome sequence information is also likely to lead to an improved understanding of the molecular mechanisms of inherited human disease, as many proteins implicated in such conditions have microbial equivalents [40-42]. The study of the physiological role of these conserved proteins will facilitate the understanding of the corresponding human disease and may help to predict the function of genes mutated in human disease states. There are certainly many other, as yet unidentified, microbial proteins with homologues in human disease, and the study of these will lead ultimately to improvements in the diagnosis and therapy of these conditions [43]. Therefore, it is possible that as well as providing information on microbial pathogenesis and identifying potential antimicrobial targets, microbial genome sequences may also contribute to our knowledge of normal gene function in man and give clues to the molecular basis of human hereditary disease.

I am grateful to Dr T. Gaasterland of the Argonne National Library for permission to use information from the MAGPIE project web site and to Dr A. Labigne for helpful discussion during the preparation of this article. P.J.J. is supported by a fellowship from the Wellcome Trust.

\section{References}

1. Sanger F, Air GM, Barrell BG et al. Nucleotide sequence of bacteriophage phi X174 DNA. Nature 1977; 265: 687-695.

2. Sanger F, Coulson AR, Hong GF, Hill DF, Petersen GB. Nucleotide sequence of bacteriophage lambda DNA. $J \mathrm{Mol}$ Biol 1982; 162: 729-773.

3. Bankier AT, Beck S, Bohni R et al. The DNA sequence of the human cytomegalovirus genome. DNA Seq 1991; 2: 1-12.

4. Goebel SJ, Johnson GP, Perkus ME, Davis SW, Winslow JP, Paoletti E. The complete DNA sequence of vaccinia virus. Virology 1990; 179: 247-266.

5. Massung RF, Esposito JJ, Liu L et al. Potential virulence determinants in terminal regions of variola smallpox virus genome. Nature 1993; 366: 748-751.

6. Adams MD, Kelly JM, Gocayne JD et al. Complementary DNA sequencing: expressed sequence tags and human genome project. Science 1991; 252: 1651-1656.

7. Adams MD, Dubnick M, Kervalage AR et al. Sequence identification of 2,375 human brain genes. Nature 1992; 355:
$632-634$

8. Fleischmann RD, Adams MD, White $\mathrm{O}$ et al. Whole-genome random sequencing and assembly of Haemophilus influenzae Rd. Science 1995; 269: 496-512.

9. Davies JE. Redundant genome sequencing? Science 1996; 273: 1155 .

10. Strauss EJ, Falkow F. Microbial pathogenesis: genomics and beyond. Science 1997; 276: 707-712.

11. Hood DW, Deadman ME, Allen T et al. Use of the complete genome sequence information of Haemophilus influenzae strain Rd to investigate lipopolysaccharide biosynthesis. Mol Microbiol 1996; 22: 951-965.

12. Hood DW, Deadman ME, Jennings MP et al. DNA repeats identify novel virulence genes in Haemophilus infuenzae. Proc Natl Acad Sci USA 1996; 93: 11121-11125.

13. Hacker J, Blum-Oehler G, Mühldorfer I, Tschäpe H. Pathogenicity islands of virulent bacteria: structure, function and impact on microbial evolution. Mol Microbiol 1997; 23: 1089-1097.

14. Mushegian AR, Koonin EV. A minimal gene set for cellular life derived by comparison of complete bacterial genomes. Proc Natl Acad Sci USA 1996; 93; 10268-10273.

15. Tomb J-F, White O, Kerlavage AR et al. The complete genome sequence of the gastric pathogen Helicobacter pylori. Nature 1997; 388: $539-547$.

16. Blattner FR, Plunkett G, Block CA et al. The complete genome sequence of Escherichia coli K-12. Science 1997; 277: 1453-1462.

17. Fraser CM, Gocayne JD, White $\mathrm{O}$ et al. The minimal gene complement of Mycoplasma genitalium. Science 1995; 270: 397-403.

18. Bult CJ, White O, Olsen GJ et al. Complete genome sequence of the methanogenic archaeon, Methanococcus jannaschii. Science 1996; 273: 1058-1073.

19. Hensel M, Shea JE, Gleeson C, Jones MD, Dalton E, Holden DW. Simultaneous identification of bacterial virulence genes by negative selection. Science 1995; 269: 400-403.

20. Shea JE, Hensel M, Gleeson C, Holden DW. Identification of a virulence locus encoding a second type III secretion system in Salmonella typhimurium. Proc Natl Acad Sci USA 1996; 93: 2593-2597.

21. Mahan MJ, Slauch JM, Mekalanos JJ. Selection of bacterial virulence genes that are specifically induced in host tissues. Science 1993; 259: 686-688.

22. Wang J, Mushegian A, Lory S, Jin S. Large-scale isolation of candidate virulence genes of Pseudomonas aeruginosa by in vivo selection. Proc Natl Acad Sci USA 1996; 93: 10434-10439.

23. Wang J, Lory S, Ramphal R, Jin S. Isolation and characterization of Pseudomonas aeruginosa genes inducible by respiratory mucus derived from cystic fibrosis patients. Mol Microbiol 1996; 22: 1005-1012.

24. Valdivia RH, Falkow S. Bacterial genetics by flow cytometry: rapid isolation of Salmonella typhimurium acid-inducible promoters by differential fluorescence induction. Mol Microbiol 1996; 22: 367-378.

25. Buchmeier NA, Heffron F. Induction of Salmonella stress proteins upon infection of macrophages. Science 1990; 248: $730-732$.

26. McGowen CC, Necheva AS, Cover TL, Blaser MJ. Acidinduced expression of oxidative stress protein homologs in Helicobacter pylori. In: Abstracts of the 97th General Meeting of the American Society for Microbiology 1997. Washington, DC, American Society for Microbiology. 1997: 38.

27. McGowan CC, Cover TL, Blaser MJ. Helicobacter pylori and gastric acid: biological and therapeutic implications. Gastroenterology 1996; 110: 926-938.

28. Liang $P$, Pardee AB. Differential display of eukaryotic messenger RNA by means of the polymerase chain reaction. Science 1992; 257: 967-971.

29. Wong KK, McClelland M. Stress-inducible gene of Salmonella typhimurium identified by arbitrary primer PCR of RNA. Proc Natl Acad Sci USA 1994; 91: 639-643.

30. Lockhart DJ, Dong H, Byrne MC et al. Expression monitoring by hybridization to high-density oligonucleotide arrays. Nature Biotech 1996; 14: 1675-1680.

31. Southern EM. DNA chips: analysing sequence by hybridization to oligonucleotides on a large scale. Trends Genet 1996; 12: $110-115$. 
32. Fodor SPA, Read JL, Pirrung MC, Stryer L, Lu AT, Solas D. Light-directed, spatially addressable parallel chemical synthesis. Science 1991; 251: 767-773.

33. Southern EM, Maskos U, Elder JK. Analyzing and comparing nucleic acid sequences by hybridization to arrays of oligonucleotides: evaluation using experimental models. Genomics 1992; 13: 1008-1017.

34. Schena M, Shalon D, Davis RW, Brown PO. Quantitative monitoring of gene expression patterns with a complementary DNA microarray. Science 1995; 270: 467-470.

35. Pease AC, Solas D, Sullivan EJ, Cronin MT, Holmes CP, Fodor SPA. Light-generated oligonucleotide arrays for rapid DNA sequence analysis. Proc Natl Acad Sci USA 1994; 91: 5022-5026.

36. Shoemaker DD, Lashkari DA, Morris D, Mittmann M, Davis RW. Quantitative phenotypic analysis of yeast deletion mutants using a highly parallel molecular bar-coding strategy. Nat Genet 1996; 14: 450-456.

37. Kozal MJ, Shah N, Shea $\mathrm{N}$ et al. Extensive polymorphisms observed in HIV-1 clade B protease gene using high-density oligonucleotide arrays. Nature Med 1996; 2: 753-759.

38. Cronin MT, Fucini RV, Kim SM, Masino RS, Wespi RM, Miyada CG. Cystic fibrosis mutation detection by hybridization to light-generated DNA probe arrays. Human Mutat 1996; 7: $244-255$.

39. Hacia JG, Brody LC, Chee MS, Fodor SPA, Collins FS. Detection of heterozygous mutations in BRCAl using high density oligonucleotide arrays and two-colour fluorescence analysis. Nat Genet 1996; 14: 441-447.

40. Tugendreich S, Bassett DE, McKusick VA, Boguski MS, Hieter P. Genes conserved in yeast and humans. Hum Mol Genet 1994; 3: 1509-1517.

41. Bassett DE, Boguski MS, Hieter P. Yeast genes and human disease. Nature 1996; 379: 589-590.

42. Mushegian AR, Bassett DE, Boguski MS, Bork P, Koonin EV. Positionally cloned human disease genes: patterns of evolutionary conservation and functional motifs. Proc Natl Acad Sci USA 1997; 94: $5831-5836$.

43. Oliver SG. From DNA sequence to biological function. Nature 1996; 379: 597-600. 